\title{
On Asymptotic Proximity of Distributions
}

\author{
Youri Davydov • Vladimir Rotar
}

Published online: 7 August 2008

(C) Springer Science+Business Media, LLC 2008

\section{Erratum to: J Theor Probab DOI 10.1007/s10959-008-0178-2}

On the third page of this article there are a few necessary corrections to reference citations:

In the third line from the top of the page, the correct reference for the survey by Rotar (cited in the original article as reference 15 twice in this line) is the following:

Rotar, V.I.: On summation of independent variables in the nonclassical situation. Russ. Math. Surv. 37(6), $151-175(1982)$

In the seventh line from the top of the page, the reference by Götze and Tikhomirov [8] requires an additional reference citation as follows:

Gotze, F., Tikhomirov, A.: Asymptotic distribution of quadratic forms and applications. J. Theor. Probab. 15(2), 423-475 (2002)

The online version of the original article can be found under doi:10.1007/s10959-008-0178-2.

Y. Davydov (凶)

Laboratoire Paul Painlevé-UMR 8524, Université de Lille I-Bat. M2, 59655 Villeneuve d'Ascq, France

e-mail: youri.davydov@univ-lille1.fr

V. Rotar

Department of Mathematics and Statistics, San Diego State University, San Diego, CA 92182-7720, USA

e-mail: vrotar@math.ucsd.edu

V. Rotar

Central Economics and Mathematics Institute, Russian Academy of Sciences, Nakhimovsckii prospect 47, Moscow 117418, Russia 